\title{
Information gathering externalities in product markets ${ }^{1}$
}

\author{
Heski Bar-Isaac \\ New York University
}

\author{
Guillermo Caruana \\ CEMFI
}

May, 2007

\author{
Vicente Cuñat \\ Universitat Pompeu Fabra
}

\begin{abstract}
Goods and services vary along a number of dimensions independently. Customers can choose to acquire information to assess the quality of some dimensions and not others. Their choices affect firms' incentives to invest in quality and so lead to indirect externalities in consumers' choices. We illustrate these ideas in a simple model with a monopolist selling a product with two characteristics, investment in quality with stochastic realizations, and heterogeneous consumers. Consumers in choosing which information to acquire do not consider the effects on firm investment incentives and so there are indirect externalities in information gathering. Therefore, a fall in the cost of acquiring information, by changing the pattern of consumers' information gathering and thereby firm investment, can paradoxically reduce consumer surplus, profits, and welfare. We briefly consider a number of potential extensions and in particular, highlight a benefit of diversity in tastes.
\end{abstract}

JEL: D83, L15

When shopping for goods with multiple characteristics that are not easily observable, consumers' decisions on what to observe and whether to buy affect the type of good offered and, indirectly,

\footnotetext{
${ }^{1} \mathrm{~A}$ previous version of this paper was entitled "Diversity and demand Externalities: When cheap information harms consumers." We thank particularly helpful audiences at the 2005 NY Commuters' IO day 2006, Alicante, Chicago GSB, Kellogg School of Management, Oxford University, Southampton University, Technion, UCL, Université de Cergy-Pontoise, ESSET 2005 and 2006, IIOC 2006, CETC 2006, Mark Armstrong, Mariagiovanna Baccara, Matthew Bidwell, Alessandro Lizzeri, Meg Meyer, Volker Nocke, Laura Veldkamp and Lawrence White. Contact info: Bar-Isaac: heski@nyu.edu; Department of Economics, Stern School of Business, NYU, 44 West 4th street 7-73, NYC, NY 10012 USA; Caruana: caruana@cemfi.es; Casado del Alisal 5, 28014 Madrid, Spain; and Cuñat: vicente.cunat@upf.edu; UPF, Ramon Trias Fargas 25-27, 08005 Barcelona, Spain.
} 
other consumers' welfare. Think of a prospective student deciding on a business school. She is likely to be concerned by her expected salary on graduation and also by long-term career prospects. Business schools can tailor their programs in ways that affect these differently. Historically, students have relied on talking to alumni and on other independent research to gain relevant information. Business school guides and rankings have made it easier for students to learn average salaries on graduation but have had little effect on the information available on other dimensions. As a consequence, more students may only investigate and make decisions based on average starting salaries. This has induced business schools to tailor their programs to the rankings. As noted by popular commentators, the overall effect of this process may be detrimental for students. ${ }^{2}$ Similarly, the easy availability of Robert Parker's 100-point wine ratings — while benefiting many as "a democratic, no-nonsense way of jettisoning the elitist jargon that veils quality from the consumer" (Darlington (2005)) - has also been criticized on the grounds that "Part of the charm and beauty of wine is its idiosyncrasy, but when everybody tries to hit the same sweet spot, it's like making soda pop." (Joel Peterson quoted in Darlington (2005)).

These are just two examples of a more general phenomenon. There are many goods and services that are, by nature, multidimensional. Given that it is costly to verify all the characteristics of a good, consumers often make purchase decisions based only on partial information. ${ }^{3}$ In addition, firms have to decide whether and how much to invest in the quality of different dimensions. These decisions take into account that consumers are heterogenous in their search behaviour. As a consequence there are indirect externalities among consumers: In equilibrium, their individual choices affect firm investments and thereby other consumers. Our contribution in this paper is to highlight this mechanism, its implications for investment incentives, consumer surplus, and welfare; and in particular, to show that a drop in the costs of acquiring information can lead to a welfare loss.

We consider a market in which a monopolist sells a good with two characteristics. The quality of each characteristic follows an uncertain realization. Before deciding whether or not to buy the

\footnotetext{
${ }^{2}$ Schatz (1993), for example, states that: "the rankings resulting from the surveys are too one-dimensional to be taken seriously. Most likely, no single MBA program is best for everyone, and almost every program is best for someone. The match has to be individualized." (See also Education \& Social Science Library (2005) for a wideranging survey of critiques of school rankings.) In itself this observation need not imply adverse welfare consequences; however, Schatz argues that: "people foolishly tend to believe that there is significance to the order in which the schools appear". Our model assumes that all agents are fully rational but that information is costly. Without having to assume that people are foolish, we show that the rankings are relevant but, through their easy availability, can have adverse consequences.

${ }^{3}$ As discussed for example in Shugan (1980).
} 
good customers can decide to observe either quality at a cost. Meanwhile, the firm can invest to improve the likelihood of a high quality realization. We show that a fall in the cost of assessing one of the characteristics affects welfare and in particular, it may be detrimental. Underlying this result is the observation that there are inherent indirect externalities in consumers' choices of which characteristic to assess. ${ }^{4}$ A simple revealed preference argument guarantees that if the firm's behaviour is fixed then a fall in the costs of assessing one characteristic unambiguously raises consumers' welfare. This is not the case when the firm can vary its investments in response to the fall in search costs. Consumers' expected choices affect the producer's investments in the quality provision of each of the dimensions. However, customers cannot commit to their choices and do not take into account their cumulative effect on the firm's behaviour. ${ }^{5}$

Specifically, changes in aggregate consumer behaviour affect welfare in two different ways.

First, a customer may assess a dimension, choose to buy the product and, on consuming, find herself enjoying quality on the other dimension that she had not assessed. This quality results from firm investments that cater to customers who assess that other dimension. Through this externality, greater diversity in consumer behaviour increases welfare.

The second effect has an opposite implication. A customer may assess a dimension in which quality is not realized and consequently choose not to purchase, even though the firm has produced quality on the other unassessed dimension to attract a different type of buyer. This quality, which is produced but not consumed, suggests that diversity in consumer behaviour may also have a negative effect on welfare.

The effect of a fall in the customers' assessment costs on the diversity of consumer behaviour depends on the initial conditions. Suppose first that most consumers are assessing one characteristic, then a fall in the cost of assessing the other characteristic would lead to greater diversity in consumer behaviour. Alternatively, if initially most consumers assess the second characteristic, then this fall in costs reduces diversity.

\footnotetext{
${ }^{4}$ Strictly speaking these are quasi-externalities as they arise from anticipated equilibrium behaviour.

${ }^{5} \mathrm{~A}$ similar effect is at play in Varian's (1980) model of informed and uninformed consumers, where the presence of informed customers leads to lower prices for all consumers. In our model, however, the decision to get informed plays a central role and, moreover, in our multi-attribute environment consumers are simultaneously informed with respect to one characteristic and uninformed with respect to another. Thus in particular, and as discussed in Section 3.1, our model can lead to a benefit of diversity. Also note that while Varian's informed consumers lead to higher consumer surplus they lead to lower profits, in our environment (endogenous) changes to consumer information acquisition behaviour through changes to information acquisition costs can lead to non-monotonic effects on both profits and consumer surplus.
} 
Overall, the effect of a fall in search costs on welfare is ambiguous. Such a fall can either reduce or increase the diversity of consumer behaviour which, in turn, can either increase or reduce welfare.

The papers most closely related in spirit to this one are Dranove and Satterthwaite (1992), which presents a theoretical framework, and Dranove et al. (2001), which empirically highlights negative welfare consequences of quality "report cards" on physicians. These papers assume that prior to any search or assessment consumers are homogeneous (so that diversity can not be addressed and externalities between different types of consumers are not explicitly discussed). Our focus is on consumers who play an active role when choosing which information to acquire, and since consumers are different they make different choices. In particular, we highlight how their individual decisions affect each other.

A few papers have considered the possibility that some features of a good are more salient than others (Ellison, 2005; and Gabaix and Laibson, 2006). Related papers in contract law, for example Korobokin (2003) and Gilo and Porat (2006) also discuss consumers' ability to understand some details of a contract better than others. These papers tend to assume that salience is exogenous and, often, that consumers are naive in their beliefs on non-salient aspects. In contrast in our model, saliency is the result of the endogenous decisions of fully rational consumers.

This work is also related to the multi-tasking literature, for example Holmström and Milgrom (1994), but there are crucial differences. They show that in a moral hazard multi-task environment it is difficult to provide strong and well-balanced incentives. If efforts are substitutes in the cost function, strengthening incentives in one dimension leads to lower effort on the other. The driving forces in our paper are different since we do not assume substitution in the cost of effort. ${ }^{6}$ In addition, in contrast to their mechanism design approach, we analyze a situation in which firm's incentives arise endogenously from individual consumers decisions in a market setting; as such, we study an incomplete contract setting, where both parties lack important commitment abilities.

Several papers have highlighted other mechanisms through which allowing for more information can be damaging for welfare. Schlee (1996) shows that making more information publicly available might also benefit strategic rivals. ${ }^{7}$ Kessler (1998) illustrates that committing to an information structure can change the resulting choice of an explicit contract. ${ }^{8}$ Meuer and Stahl (1994) show that

\footnotetext{
${ }^{6}$ Indeed, the model delivers the result considering an investment decision in only one dimension.

${ }^{7}$ See also Sakai (1985), Gal-Or (1988), Mirman et al. (1994) and Harrington (1995).

${ }^{8}$ Building on similar intuitions arising in Cremer (1995) and Dewatripont and Maskin (1995).
} 
in a competitive market better information might lead to greater differentiation, thereby softening price competition to the detriment of consumers. Finally, Glazer and McGuire (2005) show that revealing a quality index rather than its constituents can in effect reduce the firm's feasible choices in such a way as to counter-act monopoly power.

\section{Exogenous Prices}

We first introduce a simple model in which prices are treated as exogenous. This model provides many intuitions for the rest of the paper and is later embedded within the main model of Section 2 .

Consider a monopoly producer of a single complex good, which is exogenously priced at $p$. This good has two different characteristics or dimensions ( $a$ and $b$ ). Every unit of the good can be of high or low quality in each of its two dimensions. The effective quality realization of each of them is independent and stochastic. ${ }^{9}$ For one of the characteristics the probability of high quality is fixed, but for the other it depends on the firm's investment. ${ }^{10}$ Consumers incur costs in determining the quality of an attribute before making a purchase decision.

Timing is as follows:

1. The firm invests $x$ in the quality of attribute $b$.

2. Each customer, without observing the firm investment decision, chooses to assess the quality realization on none, one, or the other attribute.

3. Each customer chooses whether or not to buy the good.

Specifically we make the following assumptions with respect to the firm and consumers:

The firm invests $x \geq 0$ in the quality of attribute $b$ at a cost of $\frac{x^{2}}{4}$. This is a sunk cost that is incurred regardless of the number of sales. The marginal cost of production is 0. Production leads to stochastic quality realizations, where $q_{i} \in\{0,1\}$ denotes the quality realization of the good in attribute $i \in\{a, b\}$. While the probability of high quality, $q_{a}=1$, in attribute $a$ is exogenous, for attribute $b$ it depends on the firm's investment. Specifically, we suppose that $\operatorname{Prob}\left(q_{a}=1\right)=\frac{1}{2}$

\footnotetext{
${ }^{9}$ The stochastic realization can be viewed either as reflecting the nature of the production technology or as reflecting idiosyncratic consumer tastes. In this latter case, higher investments in quality cater to a wider range of such tastes.

${ }^{10}$ It is reasonable to think that firms choose how much to invest in each of the dimensions. We relax this simplifying assumption and discuss some of its implications in Section 3.1.
} 
and $\operatorname{Prob}\left(q_{b}=1\right)=x$. Note that stochastic realizations of quality give consumers some incentives for assessing it.

There is a continuum of ex-ante heterogeneous consumers indexed by $t$, where $t$ is uniformly distributed on $[0,1]$. Consumer $t$ when buying one unit of the good with quality $q_{a}$ on attribute $a$ and $q_{b}$ on attribute $b$ at a price $p$ gets a utility of $t q_{a}+(1-t) q_{b}-p$. That is, all consumers value both dimensions of the good, but consumers with a high $t$ give more importance to attribute $a$ while consumers with a low $t$ give more importance to attribute $b .{ }^{11}$

Consumers cannot directly observe the quality of a good in each dimension; however, they can incur search costs to assess the quality realization of either dimension. In particular, by incurring a cost $A$, a consumer can perfectly determine whether the quality on dimension $a$ is high or low, and by incurring a cost $B$ she can perfectly learn the quality on dimension $b$.

We suppose that the consumer can search either in one dimension or the other. She may also choose to buy without assessing quality or choose to neither assess quality nor buy. We preclude the possibility of assessing both dimensions before making a decision; though, as discussed in Section 3.2 , allowing this would not overturn our qualitative results.

If the realization of the quality and the consumer's assessment behaviour leads the consumer to purchase a good of quality $\left(q_{a}, q_{b}\right)$ at the price $p$, her overall utility can be written as:

$$
U_{t}=t q_{a}+(1-t) q_{b}-p-A I_{s=a}-B I_{s=b}
$$

where $I_{s=a}$ takes the value 1 if and only if the consumer assessed dimension $a$ and takes the value 0 otherwise; $I_{s=b}$ is similarly defined. If the consumer does not buy the good, her utility is simply

$$
U_{t}=-A I_{s=a}-B I_{s=b}
$$

We now move on to characterize the equilibrium search strategies of consumers and the firm's quality investments.

\footnotetext{
${ }^{11}$ We present a model in which consumers are heterogeneous in tastes. Similarly one could think of consumers with homogeneous tastes who are heterogeneous in their search costs.
} 


\subsection{Equilibrium analysis}

We characterize the optimal behaviour, first of consumers then of the firm and bring these together to determine equilibrium.

\subsubsection{Consumers}

In general, a consumer of type $t$ has four choices: assessing characteristic $b$, assessing characteristic $a$, buying without assessing, and simply not buying the good. ${ }^{12}$ Among these four alternatives, she chooses whichever gives highest utility. The fraction of consumers who assess characteristic $b$ is crucial for determining the firm's behaviour. Suppose that there is some consumer $T$ who is indifferent between assessing $b$ and the best of her other choices. Assuming that $T$ is interior it is implicitly defined by the equation:

$$
x\left(1-T+\frac{1}{2} T-p\right)-B=\max \left\{\frac{1}{2}(T+(1-T) x-p)-A, \frac{1}{2} T+(1-T) x-p, 0\right\} .
$$

Given that the utilities of all four options are linear in $t$, it is easy to see that all consumers with $t<T$ prefer to search on dimension $b$ (and subsequently purchase if and only if they discover high quality on that dimension). ${ }^{13}$

Depending on the alternative to which consumer $T$ is indifferent, different sorts of equilibria may arise. In Section 2 we analyze all these cases, but in this introductory section we focus on instances in which all consumers decide to search, some on $a$ and the rest on $b$. As will become clear later, this equilibrium is the most relevant one and provides intuitions for the mechanisms at work in the model. Thus, here we assume that assessing $a$ gives the highest value among the alternatives on the right hand side of Equation (3) and we obtain:

$$
T=p(1-2 x)+x-2(B-A) .
$$

Note that the threshold $T$ can take any value between zero and one. A value $T=0$ would correspond to all agents searching on dimension $a$, while $T=1$ would correspond to all agents searching on dimension $b$.

\footnotetext{
${ }^{12}$ Note that a consumer who decides to assess one of the characteristics purchases if and only if the realization is high. Otherwise, she would be better off taking the same unconditional purchasing decision without paying the search cost.

${ }^{13}$ Similarly if an agent of type $t^{\prime}$ prefers to assess $A$ then all agents of type $t>t^{\prime}$ also prefer to assess $A$.
} 


\subsubsection{Firm}

The firm chooses an investment in quality $x$, in order to maximize profits given the anticipated equilibrium behaviour of consumers. A proportion $T$ of the consumers search on dimension $b$, buying whenever they find high quality on that dimension (which occurs with probability $x$ and so is influenced by the firm's choice of investment). The remaining $(1-T)$ consumers search on dimension $a$ and buy if and only if they find high quality (which occurs with probability $\frac{1}{2}$ ).

The firm's problem can be expressed as:

$$
\max _{x} \Pi=\left((1-T) \frac{1}{2}+T x\right) p-\frac{x^{2}}{4}
$$

The first order condition with respect to $x$ can therefore be written down as follows:

$$
T p-\frac{x}{2}=0
$$

In particular, we obtain:

$$
x=2 p T .
$$

Note that this expression, obtained under the assumption that all consumers were either assessing in dimension $a$ or $b$, holds more generally; i.e. independently of the behaviour of those consumers not assessing on $b$. This is because the only direct effect on profits of increasing quality on $b$ is to increase purchases on the mass $T$ of customers assessing this dimension.

\subsubsection{Equilibrium}

Conditions (7) and (4) determine the equilibrium values of $T$ and $x$ in terms of the exogenous parameters of the model $(A, B$ and $p)$ :

$$
\begin{aligned}
T & =\frac{2(A-B)+p}{1-2 p+4 p^{2}}, \\
x & =2 p \frac{2(A-B)+p}{1-2 p+4 p^{2}} .
\end{aligned}
$$

We can use the equilibrium values of $T$ and $x$ to compute the value of expected profits and 
consumer surplus:

$$
\begin{aligned}
\Pi & =p\left((1-T) \frac{1}{2}+T x\right)-\frac{x^{2}}{4}, \\
C S & =\int_{0}^{T}\left(x\left(1-t+\frac{t}{2}-p\right)-B\right) d t+\int_{T}^{1}\left(\frac{1}{2}(t+(1-t) x-p)-A\right) d t,
\end{aligned}
$$

where the first integral in the consumer surplus corresponds to the fraction $T$ of consumers who search on dimension $b$ and the second corresponds to the fraction of consumers who search on dimension $a$. Adding together expressions (10) and (11), and simplifying, we can express total welfare as:

$$
W=\Pi+C S=\frac{1}{4}\left(1+x-(x-T)^{2}\right)-A(1-T)-B T .
$$

The relationship between welfare and search costs is complex, as it depends not only on the direct effects of the cost of searching ( $A$ or $B$ ), but also on the aggregate search behaviour of consumers (summarized by $T$ ) and the change in quality provision by the firm $(x=2 p T)$. We characterize these different net effects in the next section.

\subsection{Comparative Statics on Assessment Costs}

Our primary interest is in the comparative statics of welfare with respect to $A$, the cost of assessing attribute $a .^{14}$

Ultimately we are interested in profits, $\Pi$, consumer surplus, $C S$, and Welfare, $W$, as defined above. However, it is useful to begin by considering the comparative statics of the endogenous parameters $T$ and $x$.

$$
\begin{aligned}
& \frac{d T}{d A}=\frac{d}{d A}\left(\frac{2(A-B)+p}{1-2 p+4 p^{2}}\right)=\frac{2}{1-2 p+4 p^{2}}>0, \\
& \frac{d x}{d A}=2 p \frac{d T}{d A}>0 .
\end{aligned}
$$

If it becomes more expensive to gather information on attribute $a$ then, all else equal, more consumers will gather information on attribute $b$ and so $T$ (and also $x$ ) will increase.

\footnotetext{
${ }^{14}$ Similar comparative statics could be applied to $B$, but given that quality investement in dimension $a$ is exogenous, this analysis is not as meaningful. Consumer surplus can be shown to be monotonically decreasing in $B$; however, profits and welfare can be non-monotonic in $B$.
} 
Now we examine the effect on firm profits:

$$
\frac{d \Pi}{d A}=\frac{\partial \Pi}{\partial T} \frac{d T}{d A}+\frac{\partial \Pi}{\partial x} \frac{d x}{d A}=\frac{\partial \Pi}{\partial T} \frac{d T}{d A},
$$

since $\frac{\partial \Pi}{\partial x}=0$, as $x$ is chosen to maximize the firm's profits. Thus,

$$
\frac{d \Pi}{d A}=p\left(x-\frac{1}{2}\right) \frac{d T}{d A}
$$

Given that $\frac{d T}{d A}>0$, it follows that $\frac{d \Pi}{d A}>0$ if and only if $x>\frac{1}{2}$. This result captures the inefficiency of quality produced but not consumed: a rise in the cost of assessing $a$ drives more consumers to assess characteristic $b$, this increases firm profits whenever high quality is more likely to be realized on characteristic $b$ (that is where $x>\frac{1}{2}$ ), and reduces profits in the opposite case. Thus $\frac{d \Pi}{d A}$ may be either positive (when $x$ is sufficiently high, which in turn requires many consumers to be searching on dimension $b$, which in turn is likely when $A$ is high) or negative. As the equilibrium level of investment increases with $A$, overall profits may be non-monotonic in $A$.

Next turning to consumer surplus, the total derivative with respect to $A$ yields:

$$
\frac{d C S}{d A}=\frac{\partial C S}{\partial A}+\frac{\partial C S}{\partial T} \frac{d T}{d A}+\frac{\partial C S}{\partial x} \frac{d x}{d A}
$$

We consider each term of this expression in turn.

First, the direct effect of a rise in the cost of assessing characteristic $a$ is negative: for those assessing characteristic $a$ an increase in the cost of doing so reduces their utility. Analytically, $\frac{\partial C S}{\partial A}=-(1-T)$.

Next, $\frac{\partial C S}{\partial T}=0$, that is, there is no direct effect on consumer surplus of a marginal increase in $T$. All it causes is the shift of the $T$ consumer, previously assessing attribute $a$, to assess attribute $b$ instead. But this consumer was indifferent between searching on one or the other dimension, thus her welfare is not affected. ${ }^{15}$

Finally, the indirect effect of raising $A$ through the firm's investment $x$ on consumer surplus is unambiguously positive. The effect of a change in $A$ in the equilibrium investment $x$ is positive, as shown above. Given this, for those assessing $b$, there is a greater likelihood of finding quality, and

\footnotetext{
${ }^{15}$ Note that this does not imply that $\frac{d C S}{d T}=0$, since there are indirect effects through a change in the firm's investment.
} 
for those assessing $a$, there is a higher likelihood of enjoying quality on the dimension that they do not assess. More formally,

$$
\frac{\partial C S}{\partial x}=\int_{0}^{T}\left(1-\frac{t}{2}-p\right) d x+\int_{T}^{1} \frac{1}{2}(1-t) d x=T\left(\frac{1}{2}-p\right)+\frac{1}{4}>0
$$

The second integral is positive trivially. Consider the first: $\left(1-\frac{t}{2}-p\right)$ must be positive in the range $[0, T]$ for those consumers to have an incentive to search. ${ }^{16}$

Putting these elements together, overall we can write:

$$
\frac{d C S}{d A}=-(1-T)+\left(T\left(\frac{1}{2}-p\right)+\frac{1}{4}\right) \frac{d x}{d A} .
$$

Since the first term is negative and the second term is positive, in general, we cannot sign $\frac{d C S}{d A}$, and, indeed, it may be non-monotonic. Note, however, that when $T$ is close to 1 (that is most consumers are searching on attribute $b$ ), which is likely when $A$ is relatively high, the direct effect of a change in $A$ is negligible, and so consumer surplus increases in $A$. Conversely, as can readily be verified that, if most consumers are searching on dimension $a$, the direct effect dominates and consumer surplus decreases in $A$.

We can finally turn to welfare. The discussion above allows us to conclude that welfare may be non-monotonic in the cost of assessing $a$. In particular, we have argued that both profits and consumer surplus are likely to be increasing in $A$ when the cost of assessing quality on dimension $a$ is relatively high; and decreasing when $A$ is relatively low. Therefore, this also holds for welfare. We illustrate that this is indeed the case when $p=0.6$ and $B=0.05$ through the following graph.

\footnotetext{
${ }^{16}$ Note that from Equation (3) $x\left(1-\frac{t}{2}-p\right) \geq B>0$ at $t=T$ in equilibrium and and further note that is is increasing in $t$ in the range $[0, T]$.
} 
Figure 1: Welfare against assessment cost $\mathrm{A}$ at $\mathrm{p}=0.6$ and $\mathrm{B}=0.05$.

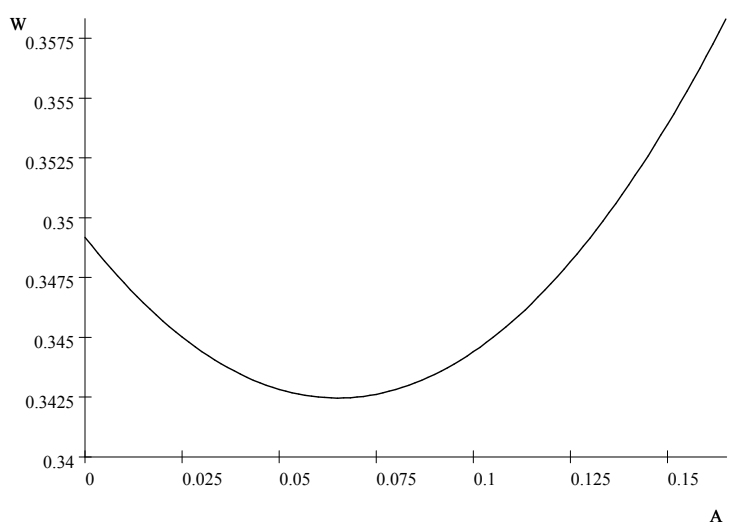

Decomposing the effect on welfare overall into the effects through firm profits and consumer surplus separately, it can further be shown that both profit and consumer surplus are non-monotonic in the cost of assessing attribute $a$; and, in particular, that both are decreasing for sufficiently low values of $A$ and increasing for high values. ${ }^{17}$

This exemplifies one of the goals of this paper: to illustrate that a reduction of the search costs can be detrimental for welfare. This is the result of a lack of coordination, or commitment, by all parties: the firm and every single consumer. If $A$ falls, all parties expect others to pay "less attention" to dimension $b$. These expectations reinforce each other and, as a consequence, in equilibrium there is both less investment by the firm and less assessment by consumers devoted to characteristic $b$. This reduces the value of the resulting good and ends up hurting everyone.

\section{Endogenous Prices}

Now we extend the analysis and allow the monopolist to choose the price it charges. In particular, we consider the following timing. First, the monopolist chooses a price $p \in \mathbb{R}$, which becomes public knowledge. Then we repeat the timing of the previous model: the firm decides on investment, which is not verifiable, and consumers take their assessment and purchase decisions.

Thus, the previous section can be seen as the equilibrium for the subgame once a particular $p$

\footnotetext{
${ }^{17}$ Specifically, here, profit takes its minimum at $A=0.001$ and consumer surplus takes its minimum at $A=0.147$.
} 
has been chosen. However, the last section only analyzed equilibria in which all consumers were searching. Here we consider all possible consumer behaviour and discuss the potential multiplicity of equilibria.

In the equilibrium analyzed in the previous section for a given price $p$, the firm exerts positive levels of investment induced by consumers assessing dimension $b$, while the others assess $a$. However, there may be other equilibria. If consumers expect less investment by the monopolist, fewer would assess dimension $b$, which makes less investment the best response for the firm. Thus, the source of multiplicity can be understood as a coordination issue: consumer expectations of high investment lead many of them to search on dimension $b$, which in turn triggers firm incentives to invest. Meanwhile low expectations have the opposite effect.

In particular, there is an equilibrium in which there is no investment. Here, for any given positive price $p$ consumers with a low $t$ (those that primarily care for dimension $b$ ) prefer not to buy. Consider other consumers. Note that the utility from buying without assessing is $\frac{t}{2}-p$ and the utility from assessing $a$ is $\frac{1}{2}(t-p)-A$; both increase linearly in $t$ at the same rate. It follows that all agents above the threshold $T^{A}=\min \{2 p, 2 A+p, 1\}$ prefer either to buy without search (when $2 p>2 A+p$ ) or (otherwise) assess $A$.

Now we turn our attention to the equilibria in which the firm invests. In all such equilibria, and following Equation (3) all agents in the interval $[0, T]$ prefer to assess characteristic $b$. In the equilibrium characterized in Section 1 the marginal consumer is indifferent between assessing either dimension $a$ or $b$. However, in principle the marginal consumer may be indifferent to not buying the good, or to buying without gathering any information. In the Appendix we provide further characterization of such equilibria. Note that consumers may vary in their behaviour in the region $(T, 1]$ and, for example, so there may be an equilibrium in which some agents assess $b$, others asses $a$, and others buy without any assessment.

In order to deal with the multiplicity of equilibria we use an equilibrium selection criterion. Before introducing it we show in the following lemma that, taking $p$ as given, there is no conflict between the interest of the firm and those of consumers.

Lemma 1 Given fixed values of $A, B$, and $p$, for any two equilibria with different investment levels, there is one that Pareto dominates the other. That is, the equilibrium with higher profits is also the one preferred by all consumers. 
Proof. Suppose that there are two equilibria 1 and 2 and denote profits, consumer surplus, quantity sold and investment by $\Pi_{i}, C S_{i}, S_{i}$ and $x_{i} i=1,2$ respectively.

First note that if $x_{1}>x_{2}$ then $C S_{1} \geq C S_{2}$. Indeed not only is aggregate consumer surplus higher, but each consumer is at least as well off. This follows since, in equilibrium 1 a consumer could deviate to behave as in equilibrium 2 , and by doing so would achieve at least the same utility level as in equilibrium 2.

Second, note that if $x_{1}>x_{2}$ then $S_{1} \geq S_{2}$. The logic here is as follows: If a given type $t$ assesses $a$ in equilibrium 2 then in equilibrium 1 she will either assess $a$ or buy without assessment; if she assesses $b$ in 2 , she will either assess $b$ or buy without assessment in 1 ; if she buys without assessment in 2 then she would do the same in 1 . In all cases, since $x_{1}>x_{2}$ sales in 2 can be no lower than sales in 1.

Finally, we show that $x_{1}>x_{2}$ implies $\Pi_{1} \geq \Pi_{2}$. Suppose for contradiction that $\Pi_{2}>\Pi_{1}$. Then in equilibrium 1, given the assessment behaviour of consumers, the firm has a profitable deviation to invest $x_{2}$. Sales under this deviation can be no lower than the sales in equilibrium 2: the investment is the same and (for the same reasons that $S_{1}>S_{2}$ before) consumers are more prone to assess and buy. Therefore deviation profits $\Pi_{D}=p S_{D}-\frac{x_{2}^{2}}{4} \geq p S_{2}-\frac{x_{2}^{2}}{4}=\Pi_{2}>\Pi_{1}$, which provides the contradiction.

We apply the following equilibrium selection criterion: Once a price is chosen, if there is more than one equilibrium consistent with it, we restrict our attention to the Pareto dominant one. ${ }^{18}$ Thus one can understand the equilibrium selection as the result of a reasonable coordination process given that, conditional on the choice of the price by the monopolist, there is no conflict of interest.

In general, and as discussed at greater length below there are four potential types of equilibria that can emerge for a given price $p$.

We refer to a Type 1 equilibrium as one in which all agents verify either one dimension or the other (this is the one analyzed in Section 1). Type 2 equilibria are the ones in which consumers either verify dimension $a$, dimension $b$ or buy without assessment. Then, there are equilibria in which there is still investment but there is a mass of consumers that do not consider buying the good (Type 4). Finally Type 3 corresponds to equilibria in which there is no investment.

\footnotetext{
${ }^{18}$ This criterion is well defined. If one compares equilibria with different investment levels, Lemma 1 shows that there is one that is Pareto dominant. Meanwhile, equilibria with the same level of investment ocurr because of consumers' indifferences. Thus, each and every consumer achieves the same utility levels in any of them, and the equilibrium chosen is the one preferred by the firm.
} 
Figure 2 shows (for the case of $A=0.05$ and $B=0.05$ ) a typical configuration of the different profits that can be achieved at different prices. The different points in the picture are equilibria when prices are fixed.

Figure 2: Firm Profits for all possible equilibria against price $\mathrm{p}$ at $\mathrm{A}=\mathrm{B}=0.05$.



At any price, there is a Type 3 equilibrium with no investment and where no consumers assess the $b$ dimension. For low prices agents with a low $t$ do not buy, while the rest buy without assessing the quality of the good (see 3a). As $p$ rises beyond 0.1 there are still some consumers who do not consider buying, but now the rest prefer to asses dimension $a$ before taking a purchase decision (3b on the figure). Finally, when $p$ is above 0.9 there is no consumer purchase or assessment in either dimension, resulting in zero profits.

When price is above 0.16 , two new equilibria can arise. The one that provides higher profits (marked as Type 1) is the one analyzed in Section 1 where all agents prefer to assess, some do so in one dimension and the rest in the other. Below it, in Figure 2 is another equilibrium (labeled as Type 4) in which there is mass of agents (those with intermediate $t^{\prime}$ s) who do not assess and do not 
buy. Note that for prices between 0.7 and 0.74 there are two possible configurations of this type of equilibrium; one with a high level of investment and a small mass of consumers that do not buy and another one with a low level of investment and a larger mass of consumers that do not buy.

The firm chooses the price that leads to the highest profit among all the possible equilibria in Figure 2. This leads to a price of 0.69 , which corresponds to a Type 1 equilibrium and to profits of 0.288. This price is the maximum that can sustain this type of equilibrium. Note that a marginal increase in price would result in some agents not buying. Given that the marginal consumer $T$ that decides not to buy is infinitesimal, the transition to the Type 4 equilibrium in terms of profits (and also investment and consumer surplus) is continuous. For $p=0.69$ there are two other equilibria coexisting with the one chosen, but both result in lower consumer surplus. Thus, both a representative consumer and the firm would prefer the same equilibrium as shown in Lemma 1.

The pattern of equilibria in Figure 2 prevails for combinations of relatively low values of $A$ and $B$. Next we fully characterize the equilibrium configurations that arise with endogenous prices for all possible combinations of the verification $\operatorname{costs} A$ and $B$. The results are summarized in Figure 3 , where the horizontal axis represents different values of $A$ and the vertical axis different values of $B$. The point marked with a star corresponds to the equilibrium depicted in Figure 3, while the horizontal line is used for the comparative statics in Section 2.1.

Figure 3: Equilibrium configuration for different values of the assessment costs A and B.

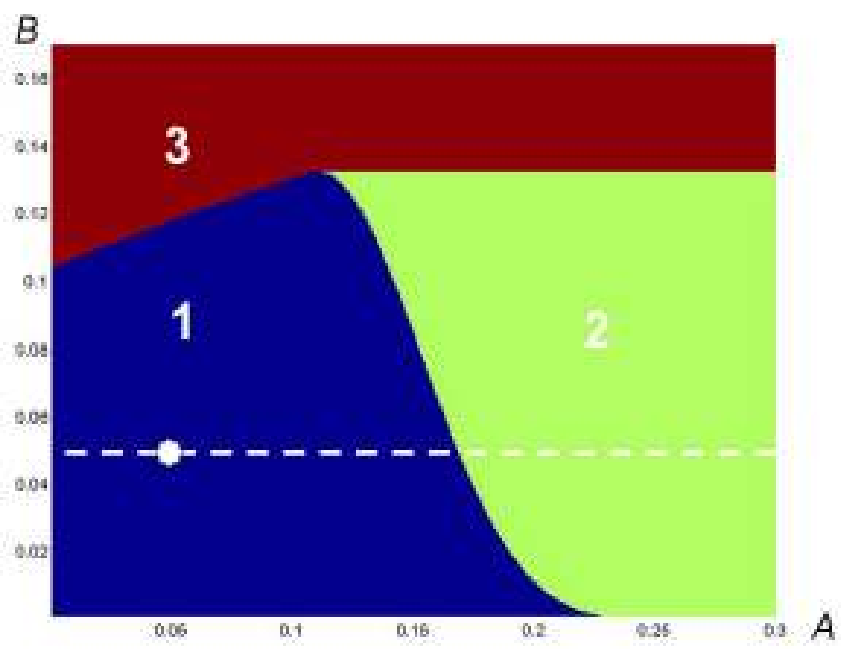


The bottom left region corresponds to equilibria of Type 1, where all agents assess either the $a$ or the $b$ dimension. When we increase the assessment cost for dimension $a$ we move to Type 2 equilibria. Some intermediate consumers buy without assessing either dimension, while those consumers very interested in only one dimension continue assessing their preferred dimension before purchase. In this region the verification costs of $a$ are high and so many consumers decide to assess dimension $b$. This gives incentives to the firm to provide high quality in $b$. This quality is sufficiently high so as to convince some consumers to buy without assessment. As $A$ grows the group of consumers that buy without verifying "moves" to the right of the distribution of agents tastes, resulting in a smaller pool of consumers assessing dimension $a$. Eventually, the cost of $A$ is high enough that no consumer assesses dimension $a$. Further increases in $A$ do not have any effect.

When $B$ is sufficiently high Type 3 equilibria (where there is no investment in quality) prevail. Here, there is no assessment in $b$ and therefore no investment. Within this area, when $A$ is low some agents do not buy and the rest assess the $a$ dimension (as in 3a of Figure 2); when $A$ is high some agents do not buy and the others buy without assessment (as in 3b of Figure 2).

Higher values of $A$ and $B$ than those depicted in Figure 3 lead to the same outcomes as the equilibria in the boundaries of the figure. At the upper boundary of the figure, no one is verifying $b$ and so further increases in $B$ have no effect. A similar argument applies for $A$.

Note that the firm can always decide not to invest, and sell to agents that value the $a$ dimension most without them assessing. As a consequence, for any $A$ and $B$ there are always sales and positive profits.

\subsection{Comparative Statics on Assessment Costs}

In this section we perform the same comparative static exercise with respect to the assessment cost $A$ that appears in Section 1.2, but now take into account the endogenous price changes. The results are shown in Figures 4 and 5. Figure 4 displays investment, price, and consumer assessment behaviour, as $A$ varies over the interval $[0,0.3]$ at $B=.05$. This corresponds to the white line of Figure 3. The transition between equilibria of Type 1 and Type 2 is marked by a dashed vertical line in each of the graphs. 
Figure 4: Comparative statics of strategies with respect to the assesment cost $\mathrm{A}$ at $\mathrm{B}=0.05$.
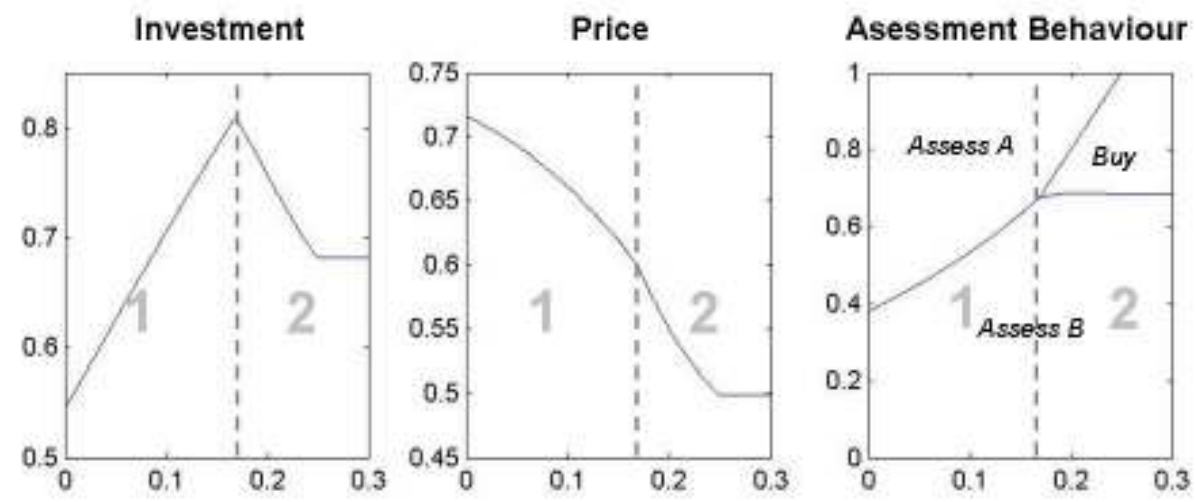

Firm investment is driven by consumers' assessment behaviour and price. For low values of $A$ all consumers assess one or other of the two dimensions (Type 1 equilibrium). As $A$ increases, fewer consumers assess dimension $a$ and more consumers search dimension $b$, causing an increase in quality provision. The effect of consumer assessment behaviour dominates the fall in price which would tend to reduce investment. When $A=0.169$ there is a regime change to Type 2 equilibria; some consumers who were searching on dimension $a$ now decide to buy without any search. Since price is falling the firm's investment falls. Once $A$ goes beyond 0.249 there are no consumers assessing dimension $a$ and therefore further increases in $A$ are irrelevant.

Price is monotonically decreasing in $A$. As the cost of assessing $a$ increases, all else equal, the utility of the marginal consumer that is indifferent between assessing $a$ or not buying falls. This means that the firm needs either to increase investment or reduce price or both to induce this consumer to continue buying with some probability. In Type 2 equilibria investment is falling with the increase in $A$ so price must necessarily fall; in Type 1 equilibria investment increases with $A$ but not enough to make price decrease.

The consumer behaviour panel shows two graphs, one represents the marginal consumer that assesses dimension $a$, and the other one the marginal consumer that assesses dimension $b$. In Type 
1 equilibria, these two coincide, while in Type 2 equilibria, there is a mass of consumers that buys without assessment.

Figure 5 shows the evolution of profits, consumer surplus and total welfare as $A$ increases at $B=0.05$.

Figure 5: Comparative statics of welfare with respect to the assesment cost $\mathrm{A}$ at $\mathrm{B}=0.05$.
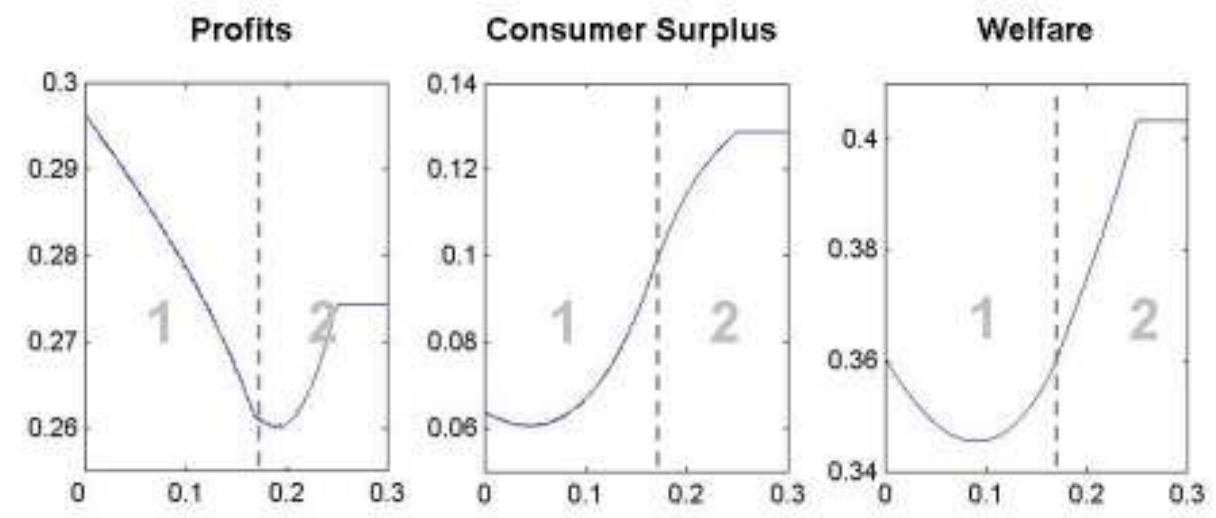

Profits are non monotonic in $A$. In the first equilibrium regime, prices are decreasing and investment is increasing. These effects dominate the contribution from any additional quantity sold. After the regime change, investment decreases in $A$, sales increase (some consumers stop assessing and always buy), but prices are still falling, however the increase in sales eventually reverses the trend of profits.

Consumer Surplus is non monotonic in $A$. When $A$ is small, a large proportion of consumers verifies dimension $a$. As $A$ increases the verification cost for these consumers increases, and given that there are many of them, this negative (direct) effect on consumer surplus dominates the positive (indirect) effects through increased investment and lower price. However, as the number of consumers that verify dimension $a$ falls the latter effects dominate the direct effect of a higher verification cost. 
Welfare is non monotonic in the verification cost $A$, this is a central result of this paper. It parallels the one presented in Section 1.2, though here prices are endogenous. Note that the non monotonicity in welfare occurs within equilibria of Type 1. That is, it is not due to a regime change. An intuition for this non-monotonicity is that as consumers start shifting attention from dimension $a$ and assess dimension $b$ instead investment increases. At some point, this compensates for the welfare loss of a higher $A$.

Individual consumer behaviour underlies this result. Consumers do not coordinate their actions and so the equilibrium may be far from the first best. Therefore, the effect of a rise in the cost of information gathering is indeterminate, and as formalized above, it does indeed have ambiguous consequences. Moreover, our analysis suggests in which circumstances the "peculiar" comparative static - that a rise in cost of assessment increases consumer surplus - would arise: When the direct effect is small (because few people are currently assessing that dimension) and the indirect effects, driving consumers to assess the other dimension and the greater investment that this entails, is large. $^{19}$

\section{Discussion and extensions}

Above, we have presented a model in which heterogeneous consumers gather information on different attributes of a good. We demonstrated a number of comparative statics results; in particular, that consumer surplus and profits can both rise with an increase in the costs of assessment. Here we consider three possible extensions. We consider exogenous prices for ease of exposition.

\subsection{Investment in both dimensions and benefits to diversity}

Extending the model to allow for investment in both dimensions does not overturn the qualitative results of the paper. ${ }^{20}$ We illustrate this through the comparison of cases (I) and (III) of Example 1. It demonstrates that profits, consumer surplus, and welfare can be non-monotonic in assessment costs.

We have shown that a fall in the cost of assessment in one dimension can lead to a decrease in

\footnotetext{
${ }^{19}$ If the firm makes investment decisions in both dimensions (the model discussed in Section 3.1) then there may be a secondary effect (through higher investment) reinforcing the direct effect. If the "peculiar" comparative static is to prevail, this effect should be relatively small.

${ }^{20}$ In fact, the model in Section 1 can be thought of as one where there are investment decisions to be made in both dimensions but where the cost of investment in dimension $B$ is $c_{b}\left(x_{b}\right)=\frac{x_{b}^{2}}{4}$, the cost of investment in dimension $A$ is a degenerate cost function (where it is costless to invest $x_{a}=\frac{1}{2}$ and infinitely costly to invest more).
} 
welfare. This is due to a fall in attention (and so investment) in the other dimension. This result is established while assuming that investing in one dimension has no direct effect on the cost of investing in the other. Allowing for substitutability of costs in investment as in Holmström and Milgrom (1990) and the multi-tasking literature, might work to exacerbate the negative effect on welfare of a fall in assessment costs.

The possibility that the firm can invest in both dimensions leads to an additional insight. Specifically, the indirect externalities driving many of the results in the model can explain a benefit of diversity in preferences. When an agent assesses dimension $b$, this benefits all other agents by raising the firm's level of investment in dimension $b$. In particular, an agent assessing dimension $a$ might be glad of the presence of an agent with different preferences who ensures some investment in dimension $b$. Indeed to the extent that her own assessment behaviour ensures "enough" investment in $a$, she might prefer that there are others in the population with preferences that induce them to assess $b$. Overall, all agents might prefer a heterogeneous population to a homogeneous one. We illustrate this possibility through cases (I) and (II) of Example 1.

This discussion relates our work to wider discussions on the benefits of diversity. Waldfogel (2003) treats this issue empirically and focuses on product proliferation with heterogeneous consumers. In his framework consumers unambiguously benefit from the presence of more consumers with similar preferences. Our example shows a contrasting result: In our setting verifying quality is costly and therefore there can be a benefit from diversity in consumer search, which arises from underlying diversity in consumer preferences. Further, many formal models in economic geography directly incorporate a benefit of diversity through complementarity in the production function or returns to specialization. Rather than highlighting the role of diversity in production, our arguments stress that there may be benefits of diversity on the demand side. That such benefits play an important role in large multi-cultural cities has been eloquently if informally suggested in Diamond et al. (2004). In other related work, Glaeser et al. (2001) have discussed the importance of amenities and the growth of "consumer cities"; our framework can provide a micro-foundation and suggests that diversity per se may play an important role.

Example 1 Suppose the firm chooses investments $x_{a}$ and $x_{b}$ in dimensions $A$ and $B$ at a cost of $\frac{1}{2}\left(x_{a}^{2}+x_{b}^{2}\right)$. These investments result in high quality with probabilities $x_{a}$ and $x_{b}$ respectively. The price of the good is 0.5 . A consumer can pay a cost 0.04 to learn the quality realization on either one 
of the two dimensions. There are two types of consumers: an $\alpha$ consumer with utility $0.55 q_{a}+0.45 q_{b}$ from consuming a good of quality $\left(q_{a}, q_{b}\right)$ and a $\beta$ consumer with utility $0.45 q_{a}+0.55 q_{b}$. There is a mass 1 of consumers. We consider three cases:

I. (benchmark) Suppose that there are equal numbers of type $\alpha$ consumers and type $\beta$ consumers. In this case there is an equilibrium in which $x_{a}=x_{b}=0.25$, the type $\alpha$ consumer assesses dimension $A$ and the type $\beta$ consumer assesses dimension $B$. In this equilibrium both consumers enjoy positive expected surplus (and the firm makes positive profits).

II. (homogeneity can harm) If all consumers are $\alpha$ type consumers (or all are $\beta$ type) the unique equilibrium is one in which no consumer assesses and there is no investment in either dimension.

III. (similar qualitative results) Next we consider comparative statics with respect to the assessment cost A. Again suppose that there are equal numbers of each type of consumer, as in the benchmark case (I). But now suppose that the cost of assessing dimension a is 0.01 while keeping $B=0.04$. Here the unique equilibrium has no investment, sales or assessment.

\subsection{Assessing both dimensions}

Hitherto we have restricted consumers from assessing both dimensions. There are a couple of ways in which one could allow them to do so. First by supposing that when the consumer assesses both characteristics she does so simultaneously. Second, by supposing that the consumer assesses sequentially, so that her decision to assess the second characteristic depends on what she has learned about the first. The latter approach makes assessing both characteristics more attractive and therefore we follow that approach in this section.

For an agent assessing characteristic $b$, conditional on doing so there is less to be gained from searching on $a$. Thus, the propensity to do so would be diminished. Even if agents had the opportunity to search on both dimensions, there would still be consumers who switch from searching on $a$ to searching on $b$; and these are the key driving force in our analysis. Equivalently, while gathering information on one characteristic does not increase the cost of gathering information on the other, it can reduce the benefit of doing so: there may be less value in learning the information on the second characteristic when knowing the realization on the first gives a great deal of information on the benefits of buying the good. ${ }^{21}$

\footnotetext{
${ }^{21}$ In particular this observation presents a further contrast with the multi-tasking literature, following Holmström
} 
Below we present a minimal example to show that allowing consumers to assess both dimensions does not invalidate our results.

Example 2 Suppose there are two types of consumer: an $\alpha$ consumer for whom $t=0.55$ and $a$ $\beta$ type consumer for whom $t=0.45$ (as in Example 1). Consumers can verify one dimension a, dimension b, both or none. There is a mass 1 of consumers, with equal numbers of each type of consumer. The firm can choose either no investment which ensures low quality in dimension $B$ or else to invest at a cost $c=0.05$ which yields high quality with probability $\frac{1}{2}$. Finally, suppose that the price is 0.45 .

If $A=B=0.04$ there is an equilibrium in which the firm invests, $\alpha$ consumers assess characteristic $A$, and $\beta$ consumers asses characteristic B. Here, consumer surplus, profits and welfare are positive.

If $B=0.04$ but $A=0.01$, the unique equilibrium is one in which there is no investment, no assessment and no sales. Clearly, there are no profits nor consumer surplus, and so everyone is worse off than with the higher assessment costs.

\subsection{Commitment to investment}

Up to this point, we have assumed that consumers do not observe the firm's investment. Although, for many applications it seems reasonable to assume that a firm cannot commit to its investment, there are examples where it may be more appropriate to suppose that consumers know the firm's investment when they take their decisions. Since investment leads to a stochastic realization on characteristic $b$, consumers still have a reason to assess before purchase. As a consequence, and given that consumers decisions are decentralized, our earlier results and considerations still apply. In particular, a rise in the cost of assessing $a$, can increase consumer surplus.

Again, just as in Section 1, we focus on the case where all agents assess. Given the firm investment $x$, the marginal consumer $T(x)$ indifferent between assessing $a$ and $b$ is characterized by:

and Milgrom $(1990,1994)$. At the heart of those results is the substitutability of effort in costs-an increase in productive effort in one dimension makes it more costly to exert effort in another. The results from the main section might suggest that our results hinge on a similar substitutability of effort but in the demand side-if a consumer is assessing characteristic $A$, it raises the costs of assessing characteristic $B$ to infinity. Although, we believe that it is interesting and noteworthy that such effects might arise on the demand as well as the supply side, this intuition and the example highlight that there is a further effect at play here. Even when assessing one characteristic does not affect the cost of assessing the other, the benefits are endogenously altered. 


$$
T(x)=p(1-2 x)+x-2(B-A) .
$$

All consumers in the range $[0, T(x)]$ assess characteristic $b$ and all other consumers assess characteristic $a$.

The firm's problem differs from that in the no-commitment case, as described in Equation (5), since the firm's investment decision affects consumer behaviour. Now, the firm's problem is:

$$
\max _{x} \Pi=\left((1-T(x)) \frac{1}{2}+T(x) x\right) p-\frac{x^{2}}{4},
$$

and so

$$
x=p(4(A-B+x+p)-1-8 p x) .
$$

Equations (22) and (20) determine equilibrium investment and consumer behaviour. ${ }^{22}$ One can then calculate consumer surplus, welfare and profits just as in the no-commitment case.

Note that $\frac{d x}{d A}=4 p>0$ and $\frac{d T}{d A}=4 p(1-2 p)+2>0$. It follows that, just as in the analysis following Equation (17), there is an indirect effect which can lead to a rise in $A$. As a result consumer surplus, profits and welfare can be non-monotonic in $A$. This is the case at $B=0.05$, and $p=0.5$, as shown in Figure 6 .

\footnotetext{
${ }^{22}$ One must verify that this is indeed an equilibrium.. That is, that all consumers prefer to assess both to not buy and buy without assessment. In fact, it is sufficient to check it for $T(x)$.
} 
Figure 6: Welfare against assesment cost $\mathrm{A}$ at $\mathrm{B}=0.05$ and $\mathrm{p}=0.5$ when the firm commits to its investment.

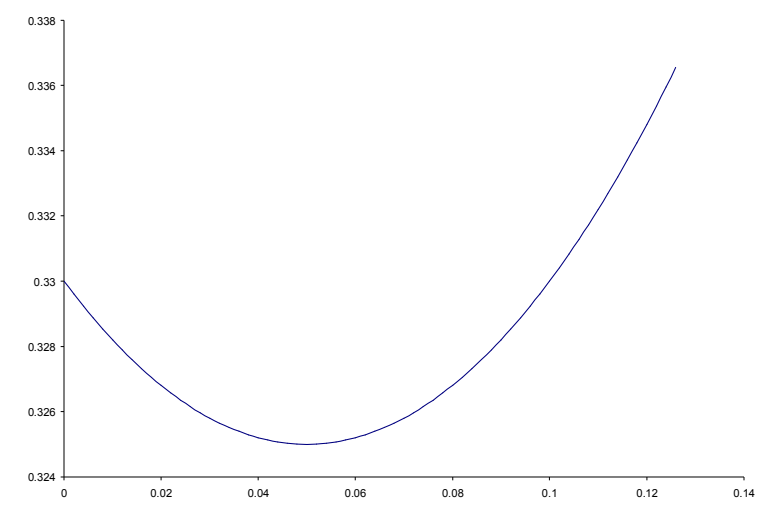

\section{Final Remarks}

The model highlights a number of effects at work in consumer markets for multi-dimensional goods where consumers can actively gather information. We have stressed, in particular, that individual's information gathering influences firm investment and thereby other consumers' welfare. Since consumers have no way of internalizing the effect of their aggregate choices on the firm's investment in quality, a change in these choices induced by a fall in the costs of information on product quality can reduce welfare. There are two effects at work. First, consumers benefit from enjoying quality on characteristics that they have not assessed; and second, high quality realizations may be left undiscovered. Our results seem to accord with the evidence that more easily available information has lead to adverse welfare effects in the context of health markets (Dranove et al (2001)), and with the related popular perception for the cases of business schools and wine rankings.

One rationale for an exogenous change in the cost of acquiring information is the appearance of intermediaries, as in the example of school rankings. While a comprehensive analysis of the incentives and industrial organization of information intermediaries is of considerable interest, it is not the goal of this paper. Nevertheless, our framework can be used to analyse the introduction of an intermediary.

In particular, even an intermediary who works on behalf of consumers may be constrained. 
First, note that by their nature some characteristics of goods have idiosyncratic appeal and are hard to describe. It is easy to describe salary on graduation from a business school, but harder to describe the extent to which the teaching style and culture will appeal. Similarly, it is easy to convey the calorific value but not the taste of food. ${ }^{23}$ In the context of our model, for goods where tastes are idiosyncratic, it may be impossible for the intermediary to enable individuals to cheaply determine whether they would enjoy a particular characteristic. Thus the intermediary might act to reduce assessment costs on some characteristics but not others, with the consequences implied by the model. It is still conceivable that an intermediary could convey the average quality of a characteristic (for example by conducting surveys). Then consumers, before further investigation, would know the expected satisfaction from that characteristic. This is analogous to Section 3.3 in which the monopolist could commit to quality investment. The key for potentially damaging effects of a fall in assessment costs is consumers' inability to commit to their information gathering behaviour (neither with respect to the firm nor to each other). An intermediary able to coordinate consumer behaviour and force commitment would overcome this, but it is hard to envisage such an intermediary as a practically feasible institution.

Interesting dynamic implications can arise if it takes time for agents to change their behaviour. In particular, a fall in the cost of acquiring information could initially raise consumer surplus and overall welfare. However, the subsequent reaction of the firm to the consumers' new search behaviour may lead to a reduction in welfare in the long run. This will be the case even when all agents are fully aware of these mechanisms due to the inability of consumers to coordinate and commit to an aggregate assessment strategy. This might explain why policies or innovations such as the introduction of school league tables in the UK, while initially welcomed are eventually reviled.

A natural extension of this paper is to consider competition. However, the analysis of competition is not trivial and lies beyond the scope of the paper. Further, we have treated the cost of acquiring information as exogenous. In related work, Bar-Isaac, Caruana and Cuñat (2007) investigate the firm's ability and desire to use marketing and advertising to highlight, or to the contrary, obfuscate product characteristics.

\footnotetext{
${ }^{23}$ This example is drawn from Scitovsky's (1976) enlightening analysis of consumer behaviour and the nature of goods.
} 


\section{References}

[1] Bar-Isaac, H. Caruana, G. and V. Cuñat (2007): "Information Gathering and Marketing," working paper

[2] Cremer, J. (1995): "Arm's Length Relationships," Quarterly Journal of Economics, 110, pp. 279-295.

[3] Darlington, D. (2005): "The Chemistry of a 90 (plus) wine," New York Times, August 7, 2005, Section 6; Column 1; Magazine Desk; pp. 36.

[4] Dewatripont, M. and E. Maskin (1995): "Contractual Contingencies and Renegotiation," RAND Journal of Economics, 26, pp. 704-719.

[5] Diamond, M., A. Horowitz and A. Yauch: (2004) "An Open Letter to NYC," To the 5 Boroughs, Capitol Records.

[6] Dranove, D., D. Kessler, M. McClellan and M. Satterthwaite (2001): "Is More Information Better? The Effects of Health Care Quality Report Cards," Journal of Political Economy, 111, pp. 555-88.

[7] Dranove, D., and M. Satterthwaite (1992): "Monopolistic Competition when Price and Quality are Imperfectly Observable," The RAND Journal of Economics, 23, pp. 518-534.

[8] Ellison, G. (2005): "A Model of Add-on Pricing," Quarterly Journal of Economics, 120(2), pp. 585-637.

[9] Education \& Social Science Library College and University Rankings: Caution and Controversy, a web-based document found on June 20, 2005 at http://www.library.uiuc.edu/edx/rankoversy.htm.

[10] Gabaix, X. and D. Laibson (2006): "Shrouded Attributes, Consumer Myopia, and Information Suppression in Competitive Markets" Quarterly Journal of Economics, 121(2), pp. 505-540

[11] Gal-Or, E. (1988): "The Advantages of Imprecise Information," RAND Journal of Economics, 19, pp. $266-275$.

[12] Gilo, D. and A. Porat (2006): "The Hidden Roles of Boilerplate and Standard-Form Contracts: Strategic Imposition of Transaction Costs, Segmentation of Consumers and Anticompetive Effects," Michigan Law Review, 104, pp. 983-1032.

[13] Glaeser, E., J. Kolko and A. Saiz (2001): "Consumer Cities," Journal of Economic Geography, 1, pp. $27-50$. 
[14] Glazer, J. and T. McGuire (2005): "The Economics of Quality Indexes," mimeo.

[15] Harrington, J. (1995): "Experimentation and Learning in a Differentiated Products Duopoly," Journal of Economic Theory, 66, pp. 275-288.

[16] Holmström, B. and P. Milgrom (1990): "Regulating Trade Among Agents," Journal of Institutional and Theoretical Economics, 146.1, pp. 85-105.

[17] Holmström, B. and P. Milgrom (1994): "The Firm as Incentive System," American Economic Review, 84, pp. 972-991.

[18] Kessler, A. (1998): "The Value of Ignorance," RAND Journal of Economics, 29, 339-354.

[19] R. Korobkin (2003): "Bounded Rationality, Standard Form Contracts, and Unconscionability," University of Chicago Law Review, 70, pp. 1203-95.

[20] Meuer, M. and D. O. Stahl II (1994): "Informative Advertising and Product Match," International Journal of Industrial Organization, 12(1), pp. 1-19

[21] Mirman, L., L. Samuelson and E. Schlee (1994): "Strategic Information Manipulation in Duopolies," Journal of Economic Theory, 62, pp. 363-384.

[22] Sakai, Y. (1985): "The Value of Information in a Simple Duopoly Model," Journal of Economic Theory, 36, pp. 36-54.

[23] Schatz, M. (1993): "What's Wrong With MBA Ranking Surveys?" Management Research News, 16, pp. 15-18.

[24] Schlee, E. (1996): "The Value of Information about Product Quality," RAND Journal of Economics, 27, pp. 803-815.

[25] Scitovsky, T. (1976): The Joyless Economy, Oxford University Press.

[26] Shugan, S. M. (1980): "The Cost of Thinking," Journal of Consumer Research, 7, pp. 99-111.

[27] Varian, H. R. (1980): "A Model of Sales," American Economic Review, 70(4), pp. 651-659.

[28] Waldfogel J. (2003): "Preference Externalities: An Empirical Study of Who Benefits Whom in Differentiated Product Markets," RAND Journal of Economics, 34, pp. 557-568.

\section{A Appendix}

Following the discussion in Section 2, there are a number of possible equilibria left to characterize. Specifically, out of Equation 3 we analyzed the case in which the marginal $T$ was indifferent between assessing dimension $b$ and dimension $a$. Now we deal with the other two possibilities, namely that (i) $T$ is indifferent between assessing dimension $b$ and not buying; and (ii) $T$ is indifferent between assessing dimension $b$ and buying without assessment. The former are Type 4 equilibria, as described in Section 2, whereas the latter 
can be either Type 2 or Type 4 equilibria (in the latter case there will be both some agents who do not consider buying and others who buy without assessment). We consider each case in turn:

(i) $T$ is determined by

$$
x\left(1-T+\frac{1}{2} T-p\right)-B=0,
$$

and $x$ is determined by Equation (7). In equilibrium, both (23) and (7) must hold and so, as long as a solution exists there are two possibilities

$$
\begin{aligned}
& T=\frac{p-p^{2}-\sqrt{-B p+p^{2}-2 p^{3}+p^{4}}}{p}, \\
& T=\frac{p-p^{2}+\sqrt{-B p+p^{2}-2 p^{3}+p^{4}}}{p} .
\end{aligned}
$$

In principle both these values, and the associated levels of investment may be interior, and so there may be two equilibria of this form, as arises, for example, in Figure 2.

Having characterized $T$, one can substitute back into Equation (7) to determine $x$. All that remains to complete the characterization of the equilibrium is to determine whether consumers in $(T, 1]$ prefer not to buy, or to assess $a$. This is a mechanical exercise for which further details are available on request.

(ii) $T$ is determined by

$$
x\left(1-T+\frac{1}{2} T-p\right)-B=\frac{T}{2}+x(1-T)-p .
$$

and, again, $x$ is determined by Equation (7). There are two possibilities:

$$
\begin{aligned}
& T=\frac{1+4 p^{2}+\sqrt{16 B p-8 p^{2}+16 p^{4}+1}}{4 p}, \\
& T=\frac{1+4 p^{2}-\sqrt{16 B p-8 p^{2}+16 p^{4}+1}}{4 p} .
\end{aligned}
$$

One can complete the characterization as in case (i). 